\title{
63 Years and 715 Days to the "Boxed Warning": Unmasking of the Propylthiouracil Problem
}

\author{
Scott A. Rivkees \\ Yale Pediatric Thyroid Center, Yale University, 464 Congress Avenue, New Haven, CT 06520, USA \\ Correspondence should be addressed to Scott A. Rivkees, scott.rivkees@yale.edu \\ Received 10 June 2010; Accepted 14 June 2010 \\ Copyright $\odot 2010$ Scott A. Rivkees. This is an open access article distributed under the Creative Commons Attribution License, \\ which permits unrestricted use, distribution, and reproduction in any medium, provided the original work is properly cited. \\ 715 days after potential problems related to PTU use in children were presented in a debate in front of the Lawson Wilkins Pediatric \\ Endocrine Society (LWPES), the US Food and Drug Administration issued a "black-box" warning about the hepatotoxicity risk \\ of the antithyroid drug propylthiouracil (PTU). This safety advisory followed the collective actions of academic societies, medical \\ publishers, the National Institutes of Health, and the FDA. Considering that surgery and radioactive iodine are the legitimate \\ treatment options for Grave Disease (GD), and are now the preferred alternative therapy in individuals who developed toxic \\ reactions to MMI, the use of PTU should now be limited to exceptional circumstances and pregnancy. Long-term PTU therapy, \\ especially in children, is not justifiable. The current advisory comes 63 years after the introduction of PTU for clinical use in 1947.
}

715 days after potential problems related to PTU use in children were presented in a debate in front of the Lawson Wilkins Pediatric Endocrine Society (LWPES), the US Food and Drug Administration issued a black-box warning about the hepatotoxicity risk of the antithyroid drug propylthiouracil (PTU) [1].

This safety advisory stated that "The United States Food and Drug Administration (FDA) has added a Boxed Warning to the label for propylthiouracil, a drug used to treat hyperthyroidism (overactive thyroid), to include information about reports of severe liver injury and acute liver failure, some of which have been fatal, in adult and pediatric patients using this medication." [1]. This safety advisory followed the collective actions of academic societies, medical publishers, the National Institutes of Health, and the FDA, all with the same goal-ending PTU-induced liver failure. This safety advisory comes 63 years after the introduction of PTU for clinical use for the treatment of hyperthyroidism in 1947.

Three years ago, a perspective advocating surgery as the primary form of definitive therapy for children with Graves' disease (GD) was published in The Journal of Clinical Endocrinology and Metabolism (JCEM) along with a counterpoint commentary on the use of radioactive iodine in GD $[2,3]$. The print discourse caught the attention of LWPES program planners.
On May 4, 2008, at the annual meeting of LWPES, a "Great Debate" was held between Dr. Melvin Grumbach of the University of California, San Francisco and Dr. Scott Rivkees of Yale University, focusing on the merits of surgery versus radioactive iodine treatment. Beforehand, the discussants agreed to comment on the risks associated with antithyroid drugs. A slide was presented stating that "Antithyroid Medication use Should not be Viewed as Trivial in Children. We need a black-box warning".

After the presentation and after the meeting, one of the discussants was made aware of what seemed to be a cluster of complications related to PTU. Girls aged 6, 8, and 14 years were described to have develop liver failure leading to liver transplantation within the previous two years. A 10-year-old girl who died of complications related to PTU more than a decade ago was brought to attention.

Following the meeting, PubMed.com searching revealed more than 40 reports of PTU-related liver failure and 10 reports of PTU-related deaths. There were five reports of PTU-related liver transplants. Of concern, $30 \%$ of cases were children. There were no reports of methimazole- (MMI)related liver failure in the pediatric population and far fewer reports of other serious adverse events for MMI than PTU. (These observations are summarized in the minutes of the BPCA hearing of October 28, 2009; see [4].) 
These concerning findings were brought to the attention of the FDA Division of Pharmacovigilance. These findings were also discussed on a Society Network of Pediatric Endocrinology (SNoPE) conference call, involving Dr. Karen Winer of the Eunice Kennedy Shriver National Institute of Child Health and Human Development (NICHD). The PTU-related adverse event data were forward to Dr. Winer and Dr. Donald Mattison, Branch Chief, Obstetric and Pediatric Pharmacology Branch (OPPB), and Director of the Best Pharmaceuticals for Children Act (BPCA). Upon review of the data, Dr. Mattison relayed concerns about a possible safely alert signal for PTU to the FDA. FDA database interrogation validated a potential safety problem.

Three weeks later, on October 28, 2008, a special workshop was held at NICHD under sponsorship of the OPPB and BPCA [4]. Meeting participants included experts in thyroidology, pediatric endocrinology, hepatology, drug epidemiology, and members of the FDA and NIH. To identify the potential number of children at risk, pharmacoepidemiology studies were performed. It was estimated that more than 2,500 children were taking PTU at the time of the hearings. (A more detailed analysis of PTU use has been recently reported [5].) FDA Adverse Event Reporting System (AERS) data revealed three pediatric deaths and 20 cases of acute severe liver injury in children of the previous 20 years. (A more detailed analysis of hepatotoxicity has been recently reported [6].) Data from the United Network of Organ Sharing (UNOS) revealed 10 liver transplants related to PTU over the proceeding 14 years, and no evidence for liver failure associated with MMI over this time. Of note, several cases of liver failure in the UNOS database were not in the FDA AERS database.

It was estimated that one child per year in the US developed PTU-induced liver failure leading transplantation or death. The risk of PTU-induced liver failure leading to transplantation was estimated to be about 1 in 2000 children. The number of children developing PTU-induced liver injury that was reversible was estimated to be about 1 in 200. In comparison, there was no risk of liver failure with MMI. Importantly, routine biochemical surveillance of liver transaminase levels and liver function was not expected to be useful in identifying children who would develop PTUinduced liver failure. Thus, the only effective way to minimize hepatotoxicity risk is to avoid PTU use.

Because of the nature of the workshop, formal specific recommendations were not made by the group. Drs. Rivkees and Mattison, though, developed pediatric-specific recommendations based on the following data.

(i) PTU should never be used as a first line treatment in children.

(ii) PTU should only be used in rare circumstances such as preparation for surgery in a patient allergic to MMI and use should be short-term.

(iii) PTU use in the children on therapy should be immediately stopped in favor of alternative therapies.

On April 9, 2009, Drs. Rivkees and Mattison published a letter in The New England Journal of Medicine (NEJM) calling attention to the BPCA hearings and detailing recommendations for discontinuation of PTU use [7]. A complementary, extensive report of the issue was simultaneously published in the International Journal of Pediatric Endocrinology [8].

Soon after publication of the NEJM letter, The Endocrine Society (TES) convened a panel of experts to address this issue. On April 15, 2009, TES issued a statement recommending that PTU not be used in children [9]. Two weeks later, the LWPES sent an alert to the members calling attention to the NEJM letter.

Recognizing that PTU-related liver toxicity was not unique to pediatric patients, a hearing was organized focusing on PTU risks in adults that was held on April 18, 2009, in Washington DC [10]. This hearing was jointly sponsored by the FDA and the American Thyroid Association (ATA). ATA representation included the Hyperthyroidism Task Force, chaired by Dr. Rebecca Bahn, of the Mayo Clinic. Considering that PTU is the antithyroid drug of choice during pregnancy, antithyroid drug use during pregnancy was a focus of the hearings.

The risk of PTU-induced liver failure leading to transplantation was estimated to be about 1 in 10,000 adults. The number of adults developing PTU-induced liver injury that was reversible was estimated to be about 1 in 100 individuals. The risk and nature of liver injury associated with MMI was estimated to be much less than that with PTU. Concern was raised that MMI may have potential teratogenic effects (aplasia cutis, choanal atresia, esophageal atresia, and other malformations); however, it was not clear if MMI birth defect risks were above background rates.

Based on the data presented, to minimize the risks of PTU to the mother and minimize the risk of MMI birth defects, the ATA Hyperthyroidism Task Force suggested that PTU use be restricted to the first trimester and MMI be used over the remainder of pregnancy. The need for additional research to define the relative risks of PTU and MMI during pregnancy was noted. A premium was placed on definitive therapy for women of childbearing years with GD, to avoid the need for antithyroid drugs during pregnancy.

Just after the April 18, 2009 meeting Drs. Cooper and Rivkees published a perspective on the risks of PTU in children and adults in JCEM at the request of the Editor-inChief further detailing PTU hepatotoxicity risks along with use recommendations [11]. Other reports were prepared for the American Association of Clinical Endocrinology [12] and the American Academy of Pediatrics [13]. The recommendations of the ATA Hyperthyroidism Task Force focusing on pregnancy were published in Thyroid a few months later [14].

Despite the publicity of associated with the NEJM letter and TES recommendations that PTU not be used in children, in early May 2009, an 11-year-old girl was transferred to the Yale Pediatric Thyroid Center and Yale-New Haven Children's Hospital with impending liver failure. Acute liver injury developed 6 weeks into PTU therapy for GD; the liver injury reversed and the girl was treated with radioactive iodine. The circumstances of the case were relayed to the FDA Division of Metabolism and Endocrinology Products and to the LWPES on May 13, 2009. 
On May 13, 2009 the LWPES issued an email alert to all members encouraging practitioners to avoid PTU use in children. On June 4, 2009, the FDA issued a widely publicized safety alert, notifying healthcare professionals of "serious risks, including liver failure and death associated with the use of PTU in adult and pediatric patients" [15]. This alert was designated the \#1 Endocrine Alert of 2009 by MedWatch.

In early June 2009, we became aware of another child in the US who developed liver failure a few months into PTU therapy; this child underwent a liver transplant. We are unaware of subsequent cases.

About one month after the April 18th meeting, the ATA was requested by the FDA to consider modification of product labeling of PTU. The ATA Hyperthyroidism Task Force submitted recommendations to the FDA in June 2009. Continuing to issue warnings, the FDA issued another PTU safety alert and posted an online informational show in August 2009 [16]. The program stated "do not use propylthiouracil in children unless they cannot take methimazole and no other treatments available" [17].

On April 21, 2010, 63 years after the drug was introduced for clinical use, the FDA "box warning" went into the label for PTU [1]. The warning included information about acute, severe, and fatal liver injury in the pediatric and adult population using this medication. FDA warnings also contained the recommendations that PTU should not be used in pediatric patients unless the patient is allergic to or intolerant of MMI and there are no other treatment options available, matching the original recommendations of Drs. Rivkees and Mattison $[7,8]$.

Considering that surgery and radioactive iodine are the legitimate treatment options for GD and are now the preferred alternative therapy in individuals who developed toxic reactions to MMI, the use of PTU in children (and adults) should now be limited to exceptional circumstances and pregnancy. Long-term PTU therapy, especially in children, is not justifiable.

715 days after "Antithyroid Medication use Should not be Viewed as Trivial in Children. We need a black-box warning" was flashed on a meeting screen, we now have one. Time and outcomes of children treated for GD will tell when we can flash "Mission Accomplished."

\section{References}

[1] Propylthiouracil, 2010, http://www.fda.gov/Safety/MedWatch /SafetyInformation/SafetyAlertsforHumanMedicalProducts/ ucm164162htm.

[2] J. A. Lee, M. M. Grumbach, and O. H. Clark, "Controversy in clinical endocrinology: the optimal treatment for pediatric Graves' disease is surgery," Journal of Clinical Endocrinology and Metabolism, vol. 92, no. 3, pp. 801-803, 2007.

[3] S. A. Rivkees and C. Dinauer, "Controversy in clinical endocrinology: an optimal treatment for pediatric Graves' disease is radioiodine," Journal of Clinical Endocrinology and Metabolism, vol. 92, no. 3, pp. 797-800, 2007.

[4] "Conference Proceeding: Hepatic Toxicity Following Treatment for Pediatric Graves' Disease Meeting," Eunice
Kennedy Shriver National Institute of Child Health and Human Development, October 2008, http://bpca.nichd .nih.gov/outreach/index.cfm.

[5] A. B. Emiliano, L. Governale, M. Parks, and D. S. Cooper, "Shifts in propylthiouracil and methimazole prescribing practices: antithyroid drug use in the United States from 1991 to 2008," Journal of Clinical Endocrinology and Metabolism, vol. 95, no. 5, pp. 2227-2233, 2010.

[6] Scott A. Rivkees and Ana Szarfman, "Dissimilar hepatotoxicity profiles of propylthiouracil and methimazole in children," Journal of Clinical Endocrinology and Metabolism, vol. 95, pp. 3260-3267, 2010.

[7] S. A. Rivkees and D. R. Mattison, "Ending propylthiouracilinduced liver failure in children," New England Journal of Medicine, vol. 360, no. 15, pp. 1574-1575, 2009.

[8] S. A. Rivkees and D. R. Mattison, "Propylthiouracil (PTU) hepatoxicity in children and recommendations for discontinuation of use," International Journal of Pediatric Endocrinology, vol. 2009, Article ID 132041, 8 pages, 2009.

[9] Society Issues Statement on Use of PTU in Children, http://www.endo-society.org/advocacy/insider/SocietyIssues StatementonUseofPTUinChildren.cfm.

[10] ATA and FDA jointly sponsor PTU meeting, http://www .thyroid.org/professionals/publications/news/09_06_02_ptu .html.

[11] D. S. Cooper and S. A. Rivkees, "Putting propylthiouracil in perspective," Journal of Clinical Endocrinology and Metabolism, vol. 94, no. 6, pp. 1881-1882, 2009.

[12] S. Rivkees, D. C. Cooper, and D. Mattison, "AACE Patient Safety-Editorials Propylthiouracil (PTU) Hepatotoxicity and Graves' Disease Therapy," http://www .aacepatientsafetyexchange.com/editorial/index.php?id=25.

[13] S. Rivkees and D. Mattison, "Recommendations for the immediate discontinuation of propylthiouracil (PTU) use in children. Section on endocrinology newsletter," American Academy of Pediatrics, vol. 19, pp. 11-13, 2009.

[14] R. S. Bahn, H. S. Burch, D. S. Cooper et al., "The role of propylthiouracil in the management of graves' disease in adults: report of a meeting jointly sponsored by the american thyroid association and the food and drug administration," Thyroid, vol. 19, no. 7, pp. 673-674, 2009.

[15] Information for Healthcare Professionals-PropylthiouracilInduced Liver Failure, http://www.fda.gov/Drugs/DrugSafety/ PostmarketDrugSafetyInformationforPatientsandProviders/ DrugSafetyInformationforHeathcareProfessionals/ucm 162701.htm.

[16] FDA Patient Safety News: Show \#89, August 2009.

[17] Liver failure with propylthiouracil, 2009, http://www .youtube.com/watch?v=HM21-BAV4kk. 